\title{
Risco cardiovascular em praticantes de musculação em academias de São Luís - MA
}

\author{
Cardiovascular risk in bodybuilders at academies in São Luís - MA
}

\author{
Andressa Pestana Brito ${ }^{1}$ \\ Orcid: http://orcid.org/0000-0002-5931-0164
}

Lucian Matheus Nascimento Sousa ${ }^{3}$

Orcid: https://orcid.org/0000-0001-8769-7821

Gabrielle Vieira da Silva Brasil ${ }^{5}$

Orcid: https://orcid.org/0000-0003-3386-5679

Flor de Maria Araújo Mendonça Silva ${ }^{7}$

Orcid: https://orcid.org/0000-0002-2796-0939

\author{
Adriana Sousa Rêgo ${ }^{2}$ \\ Orcid: http://orcid.org/0000-0002-2494-030X
}

Francilene Alves da Silva Lisbôa Ewerton ${ }^{4}$

Orcid: https://orcid.org/0000-0002-1448-9404

Maylla Luanna Barbosa Martins Bragança ${ }^{6}$

Orcid: : http://orcid.org/0000-0002-6402-3899

Janaina Maiana Abreu Barbosa ${ }^{8}$

Orcid: http://orcid.org/0000-0001-5263-6586

\begin{abstract}
Resumo
Introdução: $\mathrm{O}$ uso dos indicadores antropométricos tem crescido como forma simples e eficaz para identificar o risco de doença cardiovascular que é a principal causa de morte no mundo. Objetivo: Verificar os fatores associados aos indicadores antropométricos de risco cardiovascular em praticantes de musculação em duas academias de São Luís - MA.

Materiais e Métodos: Trata-se de um estudo transversal realizado com 302 praticantes de musculação de duas academias em São Luís - MA. A coleta de dados foi realizada nos meses de agosto e setembro de 2019. Utilizou-se um questionário socioeconômico e demográfico. Foram aferidas as medidas antropométricas. A regressão de Poisson foi realizada no programa Stata ${ }^{\circledR}$ versão 14.0.

Resultados: Dos praticantes de musculação, $52,3 \%$ eram do sexo feminino e $86,7 \%$ tinham de 20 a 39 anos de idade. Para o desfecho $C C$, ser do sexo feminino (IRR=1,10; IC=1,02-1,18), morar com companheiro (IRR=1,17; IC=1,08-127), ter tempo de exercício físico de sete a doze meses (IRR=1,14; IC=1,01-1,30), ser diabético (IRR=1,06; IC=1,24-1,69) e ter histórico de DCV na família (IRR=1,07; IC $=0,99-1,16)$ foram fatores de risco para DCV. Quanto ao desfecho RCEst, ser diabético (IRR=2,30; IC=1,34-3,93) foi fator de risco para DCV. Com relação ao desfecho $\mathrm{CP}$, ter ensino superior completo ( $\mathrm{IRR}=1,14$; $\mathrm{IC}=1,02-1,27$ ), fumar (IRR=1,12; IC=1,03-1,21), histórico de DCV na família (IRR=1,07; IC=1,00-1,15) foram fatores de risco a DCV. Quanto o indicador RCQ, ser diabético (IRR=9,47; IC =1,21-2,00) foi fator de risco a DCV.

Conclusão: Observaram-se vários fatores de riscos associados aos indicadores antropométricos de risco cardiovascular (CC, RCEst, $\mathrm{CP}$ e RCQ) em praticantes de musculação.
\end{abstract}

Palavras-chave: fatores de risco; indicadores antropométricos; doenças cardiovasculares; musculação

\footnotetext{
${ }^{1}$ Universidade CEUMA, São Luís, Maranhão, Brasil. E-mail: andressa.britto.ab@gmail.com

${ }^{2}$ Universidade CEUMA, São Luís, Maranhão, Brasil. E-mail: adricefs@yahoo.com.br

${ }^{3}$ Universidade CEUMA, São Luís, Maranhão, Brasil. E-mail: luciaaaaan@outlook.com

${ }^{4}$ Universidade CEUMA, São Luís, Maranhão, Brasil. E-mail: franewerton@globomail.com

${ }^{5}$ Universidade Aberta do SUS - UFMA, Maranhão, Brasil. E-mail: gabivsilva40@gmail.com

${ }^{6}$ Universidade Federal do Tocantins, Brasil. E-mail: mayllabmartins@gmail.com

${ }^{7}$ Universidade CEUMA, São Luís, Maranhão, Brasil. E-mails: floragyhn@gmail.com

${ }^{8}$ Universidade CEUMA, São Luís, Maranhão, Brasil. E-mails: janamaiana@hotmail.com
} 


\begin{abstract}
Introduction: The use of anthropometric indicators has grown as a simple and effective way to identify the risk of cardiovascular disease, which is the main cause of death in the world.

Objective: To verify the factors associated with anthropometric indicators of cardiovascular risk in bodybuilders in two gyms in São Luís - MA.

Materials and Methods: This is a cross-sectional study carried out with 302 bodybuilders from two gyms in São Luís - MA. Data collection was carried out in the months of August and September 2019. A socioeconomic and demographic questionnaire was used. Anthropometric measurements were taken. Poisson regression was performed using the Stata ${ }^{\circledR}$ version 14.0 program.

Results: Of the bodybuilding practitioners, $52.3 \%$ were female and $86.7 \%$ were between 20 and 39 years old. For the outcome $\mathrm{CC}$, being female $(\mathrm{IRR}=1.10 ; \mathrm{CI}=1.02-1.18)$, living with a partner $(\mathrm{IRR}=1.17$; $\mathrm{CI}=1.08-127)$, having exercise time physical activity from seven to twelve months (IRR $=1.14 ; \mathrm{CI}=$ 1.01-1.30), being diabetic (IRR = 1.06; $\mathrm{CI}=1.24-1.69$ ) and having a history of cardiovascular disease in family (IRR $=1.07 ; \mathrm{CI}=0.99-1.16$ ) were a risk factor for CVD. As for the outcome RCEst, being diabetic $(\mathrm{IRR}=2.30 ; \mathrm{CI}=1.34-3.93)$ was a risk factor for CVD. Regarding the outcome CP, having completed higher education $(\mathrm{IRR}=1.14 ; \mathrm{CI}=1.02-1.27)$, smoking $(\mathrm{IRR}=1.12 ; \mathrm{CI}=1.03-1.21)$, history of illness cardiovascular risk in the family ( $\mathrm{IRR}=1.07$; $\mathrm{CI}=1.00-1.15$ ) were a risk factor for CVD. As for the WHR indicator, being diabetic ( $I R R=9.47 ; \mathrm{CI}=1.21-2.00)$ was a risk factor for CVD.

Conclusion: There were several risk factors associated with anthropometric indicators of cardiovascular risk (WC, WHtR, CP and WHR) in bodybuilding practitioners.
\end{abstract}

Keywords: Risk factors. Anthropometric indicators. Cardiovascular diseases. Weight training.

\section{Introdução}

Devido às mudanças nos hábitos de vida nas últimas décadas, como alimentação inadequada e falta de exercício físico, as doenças crônicas não transmissíveis (DCNT) são atualmente um problema de saúde pública de maior impacto nas causas de morte ${ }^{1}$. Segundo a Organização PanAmericana de Saúde ${ }^{2}$ dentre as DCNT, as doenças cardiovasculares (DCV) são a principal causa de morte no mundo, mais de 289 mil pessoas morreram de DCV em $2019^{3}$.

O aparecimento das DCV está relacionado a diversos aspectos, nos quais os fatores de risco estão diretamente ligados como agentes causais que predispõem ao surgimento e ao desenvolvimento dessa doença $a^{4}$ Alguns desses fatores podem ser modificáveis como a hipertensão arterial sistêmica, colesterol sérico elevado, diabetes, sedentarismo, tabagismo, uso de anticoncepcional, estresse, obesidade e gordura abdominal, e outros não modificáveis como o sexo, hereditariedade e idade avançada ${ }^{5,6}$.

Identificar um ou mais desses fatores de risco, serve para discernir indivíduos propensos a desenvolver DCV, o que torna os fatores de risco cardiovascular uma ferramenta importante de prevenção e promoção da saúde ${ }^{7}$.

A utilização dos indicadores antropométricos tem crescido como forma simples e eficaz para estipular o risco de $\mathrm{DCV}^{8}$.Os indicadores antropométricos mais utilizados na avaliação do risco cardiovascular são a Circunferência da Cintura (CC), a Relação Cintura-Quadril (RCQ) e a Razão Cintura Estatura (RCEst) que pela baixa dificuldade de suas aplicações são amplamente utilizados em estudos epidemiológicos, possibilitando a identificação de indivíduos mais propensos a apresentarem excesso de adiposidade visceral e aumento do risco cardiovascular. ${ }^{9,10,11}$

Mais recentemente, outra medida de avaliação antropométrica que vem sendo utilizada é a circunferência do pescoço (CP). Segundo Preis et al. ${ }^{12}$ o depósito de gordura nessa região confere risco de $\mathrm{DCV}$, assim como a gordura visceral abdominal.

Nos últimos anos, houve aumento da prática de exercício físico, assim como a procura das pessoas por academias de ginástica. De acordo com o International 
Health, Racquet e Sportsclub Association (IHRSA), o Brasil possui mais de 34 mil academias, e cerca de 9,6 milhões de clientes $^{13}$.

Dessa forma, o interesse em estudar essa temática surgiu ao verificar o elevado número de pessoas com fatores de risco cardiovascular e também com a crescente procura por exercício físico em academias na cidade de São Luís - MA. Uma série de estudos sobre os riscos cardiovasculares tem sido realizados com extratos populacionais específicos como, por exemplo, em professores universitários, servidores da saúde e estudantes universitários, além de estudos realizados com o intuito de identificar o perfil alimentar ou avaliar o consumo de suplementos. Portanto, estudar grupos de praticantes de musculação ganha importância por afastar possíveis fatores desencadeadores de complicações à saúde e prevenir o risco de acometimentos cardiovasculares. Logo, faz-se necessário essa investigação cujo objetivo foi verificar os fatores associados aos indicadores antropométricos de risco cardiovascular em praticantes de musculação em duas academias de São Luís - MA.

\section{Materiais e Métodos}

\section{Amostra e tipo de estudo}

Trata-se de um estudo do tipo transversal analítico. A pesquisa foi realizada nos meses de agosto e setembro de 2019, com praticantes de musculação em duas academias em São Luís - MA. A amostra foi do tipo não probabilística, totalizando 302 indivíduos.

\section{Delineamento da pesquisa}

O estudo faz parte de uma pesquisa maior intitulada "Nível de ansiedade em praticantes de exercício físico e seus fatores associados", aprovado pelo Comitê de Ética em Pesquisa da Universidade CEUMA, conforme preconiza a Resolução $n^{\circ} 466$ de
12 de dezembro de 2012 do Conselho Nacional de Saúde, sob o número de parecer 3.540.161.

\section{Critérios de Inclusão e Exclusão}

Como critérios de inclusão foram selecionados indivíduos que praticavam musculação no mínimo há 3 meses, com idade de 20 a 59 anos, de ambos os sexos e que aceitaram de forma voluntária assinar o Termo de Consentimento Livre e Esclarecido (TCLE). Foram excluídos indivíduos que realizavam outro tipo de exercício físico (aula de Jump Fit, funcional, Fit dance etc.).

\section{Procedimentos}

Foi aplicado um questionário adaptado de Hilgenberg ${ }^{14}$, contendo variáveis socioeconômicas, demográficas, estilo de vida e antropométricas. O uso de suplementos também foi avaliado de acordo com o consumo (não ou sim).

$\mathrm{Na}$ avaliação antropométrica, foram aferidas as medidas de peso, altura, CC, circunferência do quadril (CQ) e $\mathrm{CP}$, e a partir desses dados foi realizado o cálculo do IMC, RCEst e RCQ.

Para aferição do peso foi utilizado uma balança digital da marca Plenna ${ }^{\circledR}$, com capacidade de $150 \mathrm{~kg}$ e sensibilidade de 100 gramas. Os entrevistados foram pesados sem tênis, com roupas de ginástica e antes de iniciarem qualquer exercício físico. Para aferição da estatura foi utilizado um estadiômetro da marca Filizola ${ }^{\circledR}$ com graduação em centímetros e com capacidade de dois metros.

Todas as circunferências foram aferidas em duplicata com auxílio de uma fita métrica não inextensível, da marca Sanny ${ }^{\circledR}$, com precisão de $0,1 \mathrm{~cm}$. A CC foi realizada na região abdominal livre ou despida e utilizado o ponto médio encontrado por meio de palpação da última costela e a crista ilíaca. Os entrevistados ficavam em pé, com o abdome relaxado, braços estendidos sob o corpo e pés unidos; 
a mensuração foi feita após expiração com a fita posicionada no plano horizontal no ponto marcado, tocando a pele e seguindo os contornos, mas sem os tecidos subjacentes ${ }^{15}$.

A CC foi expressa em centímetros (cm) e os pontos de cortes adotados para mulheres foram: sem risco para DCV $(\mathrm{CC}<$ $80 \mathrm{~cm})$, risco aumentado para DCV $(\mathrm{CC} \geq$ $80 \mathrm{~cm} \mathrm{e}<88 \mathrm{~cm})$ e risco muito aumentado para DCV $(\mathrm{CC} \geq 88 \mathrm{~cm})$ e para homens: sem risco para $\mathrm{DCV}(\mathrm{CC}<94 \mathrm{~cm})$, risco aumentado para DCV $(\mathrm{CC} \geq 94 \mathrm{~cm} \mathrm{e}<102$ $\mathrm{cm})$ e risco muito aumentado para DCV $(\mathrm{CC} \geq 102 \mathrm{~cm})^{16}$.

A CQ foi aferida no local de maior proeminência da região glútea com indivíduo utilizando roupas leves. $\mathrm{O}$ entrevistado estava em posição ereta, com os braços flexionados formando ângulo de $90^{\circ}$ e os pés juntos ${ }^{15}$. A RCQ foi obtida dividindo-se a $\mathrm{CC}$ pela $\mathrm{CQ}$, ambas em centímetros e foi considerada elevada os valores acima de 0,85 para mulheres e acima de 0,95 para homens ${ }^{17}$.

A CP foi realizada com o auxílio da fita posicionada na altura da cartilagem cricotireoidea. Nos homens com proeminência, a $\mathrm{CP}$ foi aferida abaixo da proeminência. A $\mathrm{CP}$ foi classificada conforme recomendações de Bem-Noun e Laor $^{18}$, sendo considerado como risco aumentado de DCV os homens com $\mathrm{CP}>$ $37 \mathrm{~cm}$ e as mulheres com $\mathrm{CP}>34 \mathrm{~cm}$.

A RCEst foi calculada a partir da divisão da $\mathrm{CC}(\mathrm{cm})$ pela estatura $(\mathrm{cm})$, e foram categorizados como risco cardiovascular as mulheres com valores de RCEst $\geq 0,53$ e homens RCEst $\geq 0,52^{19}$.

O IMC foi calculado pela equação: $\mathrm{IMC}=$ peso $(\mathrm{Kg}) /$ altura2 $(\mathrm{m})$. Os valores do IMC foram agrupados e classificados segundo recomendações da Organização Mundial da Saúde (OMS): $<18,4 \mathrm{Kg} / \mathrm{m}^{2}$, magreza; 18,5 a $24,9 \mathrm{Kg} / \mathrm{m}^{2}$, eutrofia; 25 a $29,9 \mathrm{Kg} / \mathrm{m}^{2}$ sobrepeso e $\geq 30 \mathrm{Kg} / \mathrm{m}^{2}$ obesidade $^{20}$.

Os dados foram analisados no programa Stata ${ }^{\circledR}$ versão 13.0. A análise descritiva das variáveis qualitativas foi descrita por frequências absolutas e relativas. Também foi realizada análise multivariada pelo método de regressão de Poisson, no qual foram incluídas no modelo múltiplo todas as variáveis independentes associadas aos quatro desfechos de interesse (CC, RCEst, CP e RCQ) com significância estatística de até $20 \%$. Para aceitação das associações investigadas no modelo final, foi adotado o valor de $\mathrm{p}<$ 0,05 .

\section{Resultados}

Dos 302 praticantes de musculação avaliados, $52,3 \%$ eram do sexo feminino, $86,7 \%$ tinham de 20 a 39 anos de idade, $64,2 \%$ viviam sem companheiro(a), $89,1 \%$ tinham ensino superior completo ou incompleto e $46,3 \%$ tinham renda familiar maior que quatro salários-mínimos. Quanto ao estilo de vida, $96 \%$ não fumavam e $50,7 \%$ relataram ingerir algum tipo de bebida alcoólica. Em relação à atividade física, 39,5\% praticavam musculação de 3 a 4 vezes por semana, $54,3 \%$ realizavam musculação por mais de um ano e 59,3\% não faziam o uso de suplemento. Quanto ao estresse, $\quad 50,7 \%$ se consideravam estressados, 59,3\% não tinham histórico de DCV na família, $96 \%$ não eram hipertensos e $99,7 \%$ sem diagnóstico de diabetes (Tabela 1).

Tabela 1: Característica socioeconômica, demográfica e estilo de vida em praticantes de musculação em duas academias de São Luís - MA, 2020.

\begin{tabular}{lcc}
\hline \multicolumn{1}{c}{ Variáveis } & n & \% \\
\hline Sexo & & \\
Masculino & 144 & 47,8 \\
Feminino & 158 & 52,3 \\
Idade & & \\
$20-39$ & 262 & 86,7
\end{tabular}


Brito AP, Rêgo AS, Lucian Sousa LMN, Ewerton FASL, Brasil GVS, Bragança MLBM, Silva FMAM, Barbosa JMA

40-59

40

13,3

Estado civil

Sem companheira

194

64,2

Com companheira

Escolaridade

Ensino Médio Completo e

10,9

Superior Completo e

Incompleto

\section{Renda}

Até um salário-mínimo

De um a dois salários-mínimos

De dois a três salários-mínimos

mínimos

Maior que quatro salários-

mínimos

\section{Fumo}

Não

Sim

Álcool

Não

Sim

\section{Frequência de Exercício}

\section{Físico}

6 vezes por semana

5 vezes por semana

3 a 4 vezes por semana

2 vezes por semana

Tempo de Exercício Físico

Menos de três meses

3 a 6 meses

7 a 12 meses

Mais de um ano

Uso de Suplemento

Sim

Estresse

Não

Sim

14949,3

DCV na família
Não

$179 \quad 59,3$

Sim

\section{Hipertensão}

Não 290

Sim 12

124

Diabetes

\begin{tabular}{lcc} 
Não & 301 & 99,7 \\
\hline
\end{tabular}

\begin{tabular}{lcc} 
Sim & 1 & 0,3 \\
\hline
\end{tabular}

$\begin{array}{lll}\text { Total } & 302 & 100\end{array}$

\section{Quanto às características} antropométricas, $55 \%$ estavam com excesso de peso, de acordo com o IMC. De acordo com a CP, $60,9 \%$ estavam sem risco para $\mathrm{DCV}$. Com relação à $\mathrm{CC}, 63,6 \%$ não tinham risco de $\mathrm{DCV}$, em relação à RCEst, $72,8 \%$ não estavam em risco e quanto à RCQ, $80,1 \%$ não tinham risco de DCV (Tabela 2). 
Tabela 2: Dados antropométricos de praticantes de musculação em duas academias de São Luís - MA, 2020.

\begin{tabular}{lcc}
\hline \multicolumn{1}{c}{ Variáveis } & n & \% \\
\hline IMC & & 45 \\
Eutrófico & 136 & 55 \\
Excesso de peso & 166 & 60,9 \\
Circunferência do pescoço & & 39,1 \\
Sem risco & 184 & \\
Com risco & 118 & 63,6 \\
Circunferência da cintura & & 36,4 \\
Sem risco & 192 & 72,8 \\
Com risco & 110 & 27,2 \\
RCEst & & \\
Sem risco & 220 & 80,1 \\
Com risco & 82 & 19,9 \\
RCQ & & 100 \\
Sem risco & 242 & 60 \\
Com risco & 302 & \\
\hline Total & & \\
\hline
\end{tabular}

IMC: Índice de Massa Corporal; RCEst: Razão Cintura Estatura; RCQ: Relação Cintura-Quadril.

Fonte: Dados da Pesquisa

$\mathrm{Na}$ análise não ajustada, para o desfecho CC, ser do sexo feminino $(\mathrm{IRR}=1,14 ; \mathrm{IC}=1,05-1,23)$, morar com companheiro $(\mathrm{IRR}=1,18 ; \mathrm{IC}=1,09-1,28)$, ter idade de 40 a 59 anos (IRR=1,11; IC $=0,99$ $1,29)$, ter renda de dois a três salários mínimos $(\mathrm{IRR}=0,85 ; \mathrm{IC}=0,68-1,07)$ e de três a quatro salários mínimos ( $\mathrm{IRR}=0,84$; $\mathrm{IC}=0,67-1,05)$, ter tempo de exercício físico de três a seis meses (IRR $=1,09$; $I C=0,98$ $1,21)$ e de sete a doze meses (IRR $=1,10$; $\mathrm{IC}=0,96-1,26)$, fazer uso de suplemento (IRR $=0,83 ; \mathrm{IC}=0,77-0,90)$, ser estressado (IRR $=1,09 ; \quad \mathrm{IC}=1,01-1,18), \quad$ diabético (IRR $=1,46 ; \quad \mathrm{IC}=1,41-1,52), \quad$ hipertenso
(IRR=1,16; IC=0,97-1,40) e ter histórico de DCV na família $(\mathrm{IRR}=1,10 ; \mathrm{IC}=1,02-1,19)$ foram fatores de risco para DCV. Quanto ao desfecho RCEst, ser do sexo feminino (IRR $=0,47 ; \quad \mathrm{IC}=0,31-0,70), \quad$ consumir bebida alcoólica $(\mathrm{IRR}=1,52 ; \mathrm{IC}=1,03-2,23)$, ter tempo de exercício físico de menos de três meses (IRR=1,58; IC $=1,01-1,26)$, fazer uso de suplemento $(\mathrm{IRR}=0,38 ; \mathrm{IC}=0,23$ 0,61 ), ser diabético (IRR $=3,71$; IC $=3,08$ 4,47) e ter histórico de doença cardiovascular na família $(\mathrm{IRR}=1,31$; $\mathrm{IC}=0,91-1,90)$ foram associados à $\mathrm{DCV}$ (Tabela 3).

Tabela 3 - Análise não ajustada da associação das características socioeconômicas, demográficas e estilo de vida de praticantes de musculação em duas academias de São Luís - MA, 2020.

\begin{tabular}{|c|c|c|c|c|c|c|c|c|c|c|c|c|}
\hline \multirow[b]{2}{*}{ Variável } & \multicolumn{3}{|c|}{$\mathbf{C C}$} & \multicolumn{3}{|c|}{ RCEst } & \multicolumn{3}{|c|}{$\mathbf{C P}$} & \multicolumn{3}{|c|}{ RCQ } \\
\hline & $\begin{array}{l}\text { IR } \\
\mathbf{R}\end{array}$ & $\begin{array}{c}\text { IC } \\
(95 \% \\
)\end{array}$ & $\begin{array}{c}\text { p- } \\
\text { valor }\end{array}$ & $\begin{array}{c}\text { IR } \\
\mathbf{R}\end{array}$ & $\begin{array}{c}\text { IC } \\
(95 \% \\
)\end{array}$ & $\begin{array}{c}\text { p- } \\
\text { valor }\end{array}$ & $\begin{array}{c}\mathbf{I R} \\
\mathbf{R}\end{array}$ & $\begin{array}{c}\text { IC } \\
(95 \% \\
)\end{array}$ & $\begin{array}{c}\text { p- } \\
\text { val } \\
\text { or }\end{array}$ & IRR & $\begin{array}{c}\text { IC } \\
(95 \% \\
)\end{array}$ & $\begin{array}{c}\text { p- } \\
\text { valor }\end{array}$ \\
\hline Sexo & & & & & & & & & & & & \\
\hline Masculino & 1 & - & 1 & 1 & - & 1 & 1 & - & 1 & 1 & - & 1 \\
\hline Feminino & 1,14 & $\begin{array}{c}(1,05- \\
1,23)\end{array}$ & $\begin{array}{c}<0,00 \\
1\end{array}$ & 0,47 & $\begin{array}{c}(0,31- \\
0,70)\end{array}$ & $\begin{array}{c}<0,00 \\
1\end{array}$ & 0,69 & $\begin{array}{c}(0,31- \\
0,07)\end{array}$ & $\begin{array}{l}<0 \\
001\end{array}$ & 0,39 & $\begin{array}{c}(0,23- \\
0,64)\end{array}$ & $\begin{array}{c}<0,00 \\
1\end{array}$ \\
\hline Estado Civil & & & & & & & & & & & & \\
\hline Sem companheiro (a) & 1 & - & 1 & - & - & - & - & - & - & 1 & - & 1 \\
\hline Com companheiro (a) & 1,18 & $\begin{array}{c}(1,09- \\
1,28)\end{array}$ & $\begin{array}{c}< \\
0,001\end{array}$ & - & - & - & - & - & - & 1,57 & $\begin{array}{c}(1,00- \\
2,46)\end{array}$ & 0,049 \\
\hline Idade (anos) & & & & & & & & & & & & \\
\hline $20-39$ & 1 & - & 1 & - & - & - & - & - & - & 1 & - & 1 \\
\hline $40-59$ & 1,11 & $\begin{array}{c}(0,99- \\
1,29)\end{array}$ & 0,054 & - & - & - & - & - & - & 1,81 & $\begin{array}{c}(1,08- \\
3,03)\end{array}$ & 0,024 \\
\hline
\end{tabular}


Brito AP, Rêgo AS, Lucian Sousa LMN, Ewerton FASL, Brasil GVS, Bragança MLBM,

Silva FMAM, Barbosa JMA

\begin{tabular}{|c|c|c|c|c|c|c|c|c|c|c|c|c|}
\hline \multicolumn{13}{|l|}{$\begin{array}{l}\text { Escolaridade } \\
\text { Ensino Médio }\end{array}$} \\
\hline $\begin{array}{l}\text { Completo e } \\
\text { Incompleto }\end{array}$ & - & - & - & - & - & - & 1 & - & 1 & 1 & - & 1 \\
\hline $\begin{array}{l}\text { Superior Completo e } \\
\text { Incompleto }\end{array}$ & - & - & - & - & - & - & 1,19 & $\begin{array}{c}(1,06- \\
1,34)\end{array}$ & $\begin{array}{c}0,0 \\
03\end{array}$ & 3,55 & $\begin{array}{c}(0,90- \\
13,9)\end{array}$ & 0,068 \\
\hline \multicolumn{13}{|l|}{ Renda } \\
\hline $\begin{array}{l}\text { Até um salário- } \\
\text { mínimo }\end{array}$ & 1 & - & 1 & - & - & - & - & - & - & - & - & - \\
\hline $\begin{array}{l}\text { De um a dois } \\
\text { salários-mínimos }\end{array}$ & 1,91 & $\begin{array}{c}(0,70- \\
1,17)\end{array}$ & 0,466 & - & - & - & - & - & - & - & - & - \\
\hline $\begin{array}{l}\text { De dois a três } \\
\text { salários-mínimos }\end{array}$ & 0,85 & $\begin{array}{l}(0,68- \\
1,07)\end{array}$ & 0,186 & - & - & - & - & - & - & - & - & - \\
\hline $\begin{array}{l}\text { De três a quatro } \\
\text { salários-mínimos }\end{array}$ & 0,84 & $\begin{array}{c}(0,67- \\
1,05)\end{array}$ & 0,134 & - & - & - & - & - & - & - & - & - \\
\hline $\begin{array}{l}\text { Maior que quatro } \\
\text { salários-mínimos } \\
\text { Álcool }\end{array}$ & 0,89 & $\begin{array}{c}(0,71- \\
1,10)\end{array}$ & 0,305 & - & - & - & - & - & - & - & - & - \\
\hline Não & - & - & - & 1 & - & 1 & 1 & - & 1 & - & - & - \\
\hline Sim & - & - & - & 1,52 & $\begin{array}{l}(1,03- \\
2,23)\end{array}$ & 0,032 & 1,10 & $\begin{array}{l}(1,01- \\
1,19)\end{array}$ & $\begin{array}{c}0,0 \\
15\end{array}$ & - & - & - \\
\hline \multicolumn{13}{|l|}{ Fumo } \\
\hline Não & - & - & - & - & - & - & 1 & - & 1 & 1 & - & 1 \\
\hline Sim & - & - & - & - & - & - & 1,27 & $\begin{array}{l}(1,09- \\
1,47)\end{array}$ & $\begin{array}{l}<0, \\
001\end{array}$ & 2,19 & $\begin{array}{c}(1,07- \\
4,47)\end{array}$ & 0,030 \\
\hline \multicolumn{13}{|l|}{ Tempo de } \\
\hline Mais de um ano & 1 & - & 1 & 1 & - & 1 & 1 & - & 1 & - & - & - \\
\hline 3 a 6 meses & 1,09 & $\begin{array}{c}(0,98- \\
1,21)\end{array}$ & 0,083 & 1,24 & $\begin{array}{c}(0,98- \\
1,21)\end{array}$ & 0,383 & 0,9 & $\begin{array}{c}(0,80- \\
1,00)\end{array}$ & $\begin{array}{c}0,0 \\
50\end{array}$ & - & - & - \\
\hline 7 a 12 meses & 1,10 & $\begin{array}{c}(0,96- \\
1,26)\end{array}$ & 0,153 & 1,28 & $\begin{array}{l}(0,96- \\
1,26)\end{array}$ & 0,410 & 0,92 & $\begin{array}{l}(0,83- \\
1,02)\end{array}$ & $\begin{array}{l}0,1 \\
28\end{array}$ & - & - & - \\
\hline Menos de três meses & 1,13 & $\begin{array}{c}(1,01- \\
1,26\end{array}$ & 0,027 & 1,58 & $\begin{array}{c}(1,01- \\
1,26\end{array}$ & 0,051 & 0,80 & $\begin{array}{c}(0,66- \\
0,98\end{array}$ & $\begin{array}{l}0,0 \\
33\end{array}$ & - & - & - \\
\hline \multicolumn{13}{|l|}{ Suplemento } \\
\hline Não & 1 & - & 1 & 1 & - & 1 & - & - & - & 1 & - & 1 \\
\hline $\operatorname{Sim}$ & 0,83 & $\begin{array}{c}(0,77- \\
0,90)\end{array}$ & $\begin{array}{c}<0,00 \\
1\end{array}$ & 0,38 & $\begin{array}{c}(0,23- \\
0,61)\end{array}$ & $\begin{array}{c}<0,00 \\
1\end{array}$ & - & - & - & 0,48 & $\begin{array}{c}(0,28- \\
0,83)\end{array}$ & $\begin{array}{c}<0,00 \\
8\end{array}$ \\
\hline \multicolumn{13}{|l|}{ Estresse } \\
\hline Não & 1 & - & 1 & - & - & - & - & - & - & 1 & - & 1 \\
\hline Sim & 1,09 & $\begin{array}{c}(1,01- \\
1,18)\end{array}$ & 0,019 & - & - & - & - & - & - & 1,43 & $\begin{array}{l}(0,90- \\
2,28)\end{array}$ & 0,123 \\
\hline \multicolumn{13}{|l|}{ Diabetes } \\
\hline Não & 1 & - & 1 & 1 & - & 1 & 1 & - & 1 & 1 & - & 1 \\
\hline Sim & 1,46 & $\begin{array}{c}(1,41- \\
1,52)\end{array}$ & $\begin{array}{c}<0,00 \\
1\end{array}$ & 3,71 & $\begin{array}{c}(3,08- \\
4,47)\end{array}$ & $\begin{array}{c}<0,00 \\
1\end{array}$ & 0,71 & $\begin{array}{c}(0,69- \\
0,74)\end{array}$ & $\begin{array}{l}<0 \\
001\end{array}$ & 2,33 & $\begin{array}{c}(3,22- \\
1,68)\end{array}$ & $\begin{array}{c}<0,00 \\
1\end{array}$ \\
\hline \multicolumn{13}{|l|}{ Hipertensão } \\
\hline Não & 1 & - & 1 & - & - & - & - & - & - & 1 & - & 1 \\
\hline Sim & 1,16 & $\begin{array}{c}(0,97- \\
1,40)\end{array}$ & 0,092 & - & - & - & - & - & - & 1,72 & $\begin{array}{l}(0,74- \\
3,98)\end{array}$ & 0,200 \\
\hline \multicolumn{13}{|l|}{$\begin{array}{l}\text { Histórico de DCV } \\
\text { na família }\end{array}$} \\
\hline Não & 1 & - & 1 & 1 & - & 1 & 1 & - & 1 & 1 & - & 1 \\
\hline Sim & 1,10 & $\begin{array}{c}(1,02- \\
1,19)\end{array}$ & 0,013 & 1,31 & $\begin{array}{c}(0,91- \\
1,90)\end{array}$ & 0,140 & 1,10 & $\begin{array}{c}(1,01- \\
1,19)\end{array}$ & $\begin{array}{c}0,0 \\
16\end{array}$ & 1,77 & $\begin{array}{l}(1,12- \\
2,80)\end{array}$ & 0,013 \\
\hline
\end{tabular}

IRR: Risco Relativo; IC: Intervalo de Confiança; DCV: Doença Cardiovascular.

Fonte: Dados da Pesquisa

Ainda na análise não ajustada, com relação ao desfecho $\mathrm{CP}$, ser do sexo feminino $\quad(\mathrm{IRR}=0,69 ; \mathrm{IC}=0,31-0,07)$, ter ensino superior completo ( $\mathrm{IRR}=1,19$; $\mathrm{IC}=1,06-1,34$ ), fumar (IRR=1,27; IC $=1,09$ $1,47)$, ter tempo de exercício físico de 
menos de 3 meses (IRR $=0,80$; IC $=0,66$ $0,98)$, de três a seis meses (IRR $=0,9$; $\mathrm{IC}=0,80-1,00)$, de sete a doze meses (IRR $=0,92 ; \mathrm{IC}=0,83-1,02)$, ser diabético (IRR $=0,71 ; \mathrm{IC}=0,69-0,74)$ e ter histórico de DCV na família $(\mathrm{IRR}=1,10 ; \mathrm{IC}=1,01-1,19)$ foram associados à $\mathrm{DCV}$ e quanto ao indicador antropométrico RCQ, ser do sexo feminino ( $\mathrm{IRR}=0,39$; $\mathrm{IC}=0,23-0,64)$, viver com companheiro (IRR $=1,57$; IC $=1,00$ 2,46), ), ter idade de 40 a 59 anos $(\mathrm{IRR}=1,81 ; \mathrm{IC}=1,08-3,03)$, ter ensino superior completo $(\mathrm{IRR}=3,55 ; \mathrm{IC}=0,90$ $13,9)$, fumar $(\mathrm{IRR}=2,19 ; \mathrm{IC}=1,07-4,47)$, fazer uso de suplemento $(\mathrm{IRR}=0,48$; IC $=$ 0,28-0,83), ser estressado (IRR $=1,43$; IC $=$ $0,90-2,28)$, diabético $(\mathrm{IRR}=2,33 ; \mathrm{IC}=3,22$ 1,68), hipertenso (IRR $=172 ; \mathrm{IC}=0,74-3,98)$ e ter histórico de DCV na família
$(\mathrm{IRR}=1,77 ; \quad \mathrm{IC}=1,12-2,80) \quad$ foram associados à DCV (Tabela 3 ).

$\mathrm{Na}$ análise ajustada, para o desfecho $\mathrm{CC}$, ser do sexo feminino (IRR=1, 10 ; $\mathrm{IC}=$ 1,02-1,18), morar com companheiro (IRR=1,17; IC=1,08-127), ter tempo de exercício físico de sete a doze meses (IRR=1,14; IC $=1,01-1,30)$, ser diabético $(\mathrm{IRR}=1,06 ; \mathrm{IC}=1,24-1,69)$ e ter histórico de DCV na família $(\mathrm{IRR}=1,07 ; \mathrm{IC}=0,99-1,16)$ foram fatores de risco para DCV e fazer uso de suplemento $(\mathrm{IRR}=0,86 ; \mathrm{IC}=0,80-0,93)$ foi fator protetor. Quanto ao desfecho RCEst, ser diabético (IRR=2,30; IC $=1,34$ $3,93)$ foi fator de risco para DCV e ser do sexo feminino $(\mathrm{IRR}=0,45 ; \mathrm{IC}=0,30-0,67) \mathrm{e}$ fazer uso de suplemento (IRR=0,37; $\mathrm{IC}=0,23-0,61)$ foram fatores protetores (Tabela 4).

Tabela 4 - Análise ajustada da associação das características socioeconômicas, demográficas e estilo de vida de praticantes de musculação em duas academias de São Luís - MA, 2020.

\begin{tabular}{|c|c|c|c|c|c|c|c|c|c|c|c|c|}
\hline \multirow[b]{2}{*}{ Variável } & \multicolumn{3}{|c|}{$\mathbf{C C}$} & \multicolumn{3}{|c|}{ RCEst } & \multicolumn{3}{|c|}{$\mathbf{C P}$} & \multicolumn{3}{|c|}{ RCQ } \\
\hline & $\begin{array}{l}\text { IR } \\
\mathbf{R}\end{array}$ & $\begin{array}{c}\text { IC } \\
(95 \% \\
)\end{array}$ & $\begin{array}{c}\text { p- } \\
\text { valor }\end{array}$ & $\begin{array}{l}\mathbf{I R} \\
\mathbf{R}\end{array}$ & $\begin{array}{c}\text { IC } \\
(95 \% \\
)\end{array}$ & $\begin{array}{c}\text { p- } \\
\text { valor }\end{array}$ & $\begin{array}{c}\text { IR } \\
\mathbf{R}\end{array}$ & $\begin{array}{c}\text { IC } \\
(95 \% \\
\quad)\end{array}$ & $\begin{array}{c}\text { p- } \\
\text { val } \\
\text { or }\end{array}$ & IRR & $\begin{array}{c}\text { IC } \\
(95 \% \\
\quad)\end{array}$ & $\begin{array}{c}\text { p- } \\
\text { valor }\end{array}$ \\
\hline \multicolumn{13}{|l|}{ Sexo } \\
\hline Masculino & 1 & - & 1 & 1 & - & 1 & 1 & - & 1 & 1 & - & 1 \\
\hline Feminino & 1,10 & $\begin{array}{c}(1,02- \\
1,18)\end{array}$ & $\begin{array}{c}<0,01 \\
2\end{array}$ & 0,45 & $\begin{array}{c}(0,30- \\
0,67)\end{array}$ & $\begin{array}{c}<0,00 \\
1\end{array}$ & 0,71 & $\begin{array}{c}(0,66- \\
0,76)\end{array}$ & $\begin{array}{l}<0, \\
001\end{array}$ & 0,35 & $\begin{array}{c}(0,21- \\
0,58)\end{array}$ & $\begin{array}{c}<0,00 \\
1\end{array}$ \\
\hline \multicolumn{13}{|l|}{ Estado Civil } \\
\hline Sem companheiro(a) & 1 & - & 1 & - & - & - & - & - & - & 1 & - & 1 \\
\hline Com companheiro(a) & 1,17 & $\begin{array}{c}(1,08- \\
1,27)\end{array}$ & $\begin{array}{c}< \\
0,001\end{array}$ & - & - & - & - & - & - & 1,37 & $\begin{array}{l}(0,87- \\
2,15)\end{array}$ & 0,164 \\
\hline \multicolumn{13}{|l|}{ Idade (anos) } \\
\hline $20-39$ & 1 & - & 1 & - & - & - & - & - & - & 1 & - & 1 \\
\hline $40-59$ & 1,04 & $\begin{array}{c}(0,93- \\
1,15)\end{array}$ & 0,433 & - & - & - & - & - & - & 1,55 & $\begin{array}{l}(0,90- \\
2,67)\end{array}$ & 0,106 \\
\hline \multicolumn{13}{|l|}{ Escolaridade } \\
\hline $\begin{array}{l}\text { Completo e } \\
\text { Incompleto }\end{array}$ & - & - & - & - & - & - & 1 & - & 1 & 1 & - & 1 \\
\hline $\begin{array}{l}\text { Superior Completo e } \\
\text { Incompleto }\end{array}$ & - & - & - & - & - & - & 1,14 & $\begin{array}{l}(1,02- \\
1,27)\end{array}$ & $\begin{array}{c}0,0 \\
15\end{array}$ & 3,08 & $\begin{array}{l}(0,86- \\
10,9)\end{array}$ & 0,082 \\
\hline \multicolumn{13}{|l|}{ Renda } \\
\hline $\begin{array}{l}\text { Até um salário } \\
\text { mínimo }\end{array}$ & 1 & - & 1 & - & - & - & - & - & - & - & - & - \\
\hline $\begin{array}{l}\text { De um a dois salários } \\
\text { mínimos }\end{array}$ & 0,92 & $\begin{array}{c}(0,72- \\
1,17)\end{array}$ & 0,514 & - & - & - & - & - & - & - & - & - \\
\hline $\begin{array}{l}\text { De dois a três salários } \\
\text { mínimos }\end{array}$ & 0,85 & $\begin{array}{l}(0,68- \\
1,07)\end{array}$ & 0,192 & - & - & - & - & - & - & - & - & - \\
\hline $\begin{array}{l}\text { De três a quatro } \\
\text { salários mínimos }\end{array}$ & 0,87 & $\begin{array}{l}(0,69- \\
1,09)\end{array}$ & 0,230 & - & - & - & - & - & - & - & - & - \\
\hline $\begin{array}{l}\text { Maior que quatro } \\
\text { salários mínimos }\end{array}$ & 0,92 & $\begin{array}{l}(0,73- \\
1,14)\end{array}$ & 0,468 & - & - & - & - & - & - & - & - & - \\
\hline
\end{tabular}


Brito AP, Rêgo AS, Lucian Sousa LMN, Ewerton FASL, Brasil GVS, Bragança MLBM,

Silva FMAM, Barbosa JMA

\begin{tabular}{|c|c|c|c|c|c|c|c|c|c|c|c|c|}
\hline Álcool & & & & & & & & & & & & \\
\hline Não & - & - & - & 1 & - & 1 & 1 & - & 1 & - & - & - \\
\hline Sim & - & - & - & 1,35 & $\begin{array}{c}(0,93- \\
1,96)\end{array}$ & 0,107 & 1,02 & $\begin{array}{c}(0,06- \\
1,10)\end{array}$ & $\begin{array}{c}0,4 \\
03\end{array}$ & - & - & - \\
\hline Fumo & & & & & & & & & & & & \\
\hline Não & - & - & - & - & - & - & 1 & - & 1 & 1 & - & 1 \\
\hline Sim & - & - & - & - & - & - & 1,12 & $\begin{array}{c}(1,03- \\
1,21)\end{array}$ & $\begin{array}{c}\mathbf{0 , 0} \\
\mathbf{0 7}\end{array}$ & 1,36 & $\begin{array}{c}(0,71- \\
2,61)\end{array}$ & 0,345 \\
\hline $\begin{array}{l}\text { Tempo de exercício } \\
\text { físico }\end{array}$ & & & & & & & & & & & & \\
\hline Mais de um ano & 1 & - & 1 & 1 & - & 1 & 1 & - & 1 & - & - & - \\
\hline 3 a 6 meses & 1,08 & $\begin{array}{c}(0,98- \\
1,19)\end{array}$ & 0,107 & 1,17 & $\begin{array}{c}(0,75- \\
1,83)\end{array}$ & 0,479 & 0,94 & $\begin{array}{l}(0,86- \\
1,03)\end{array}$ & $\begin{array}{c}0 \\
249\end{array}$ & - & - & - \\
\hline 7 a 12 meses & 1,14 & $\begin{array}{c}(1,01- \\
1,30)\end{array}$ & $\mathbf{0 , 0 3 2}$ & 1,25 & $\begin{array}{c}(0,68- \\
2,29)\end{array}$ & 0,459 & 0,98 & $\begin{array}{c}(0,91- \\
1,07)\end{array}$ & $\begin{array}{l}0,8 \\
47\end{array}$ & - & - & - \\
\hline Menos de três meses & 1,08 & $\begin{array}{c}(0,96- \\
1,21\end{array}$ & 0,157 & 1,22 & $\begin{array}{l}(0,76- \\
1,95\end{array}$ & 0,393 & 0,88 & $\begin{array}{c}(0,71- \\
1,08\end{array}$ & $\begin{array}{c}0,8 \\
47\end{array}$ & - & - & - \\
\hline Suplemento & & & & & & & & & & & & \\
\hline Não & 1 & - & 1 & 1 & - & 1 & - & - & - & 1 & - & 1 \\
\hline Sim & 0,86 & $\begin{array}{c}(0,80- \\
0,93)\end{array}$ & $\begin{array}{c}<0,00 \\
1\end{array}$ & 0,37 & $\begin{array}{c}(0,23- \\
0,61)\end{array}$ & $\begin{array}{c}<0,00 \\
1\end{array}$ & - & - & - & 0,46 & $\begin{array}{r}(0,26- \\
0,79)\end{array}$ & 0,005 \\
\hline Estresse & & & & & & & & & & & & \\
\hline Não & 1 & - & 1 & - & - & - & - & - & - & 1 & - & 1 \\
\hline Sim & 1,06 & $\begin{array}{c}(0,98- \\
1,14)\end{array}$ & 0,120 & - & - & - & - & - & - & 1,47 & $\begin{array}{c}(0,95- \\
2,27)\end{array}$ & 0,083 \\
\hline Diabetes & & & & & & & & & & & & \\
\hline Não & 1 & - & 1 & 1 & - & 1 & 1 & - & 1 & 1 & - & 1 \\
\hline Sim & 1,45 & $\begin{array}{c}(1,24- \\
1,69)\end{array}$ & $\begin{array}{c}<0,00 \\
1\end{array}$ & 2,30 & $\begin{array}{l}(1,34- \\
3,93)\end{array}$ & 0,002 & 0,62 & $\begin{array}{c}(0,57- \\
0,67)\end{array}$ & $\begin{array}{l}<0 \\
001\end{array}$ & 9,47 & $\begin{array}{l}(1,21- \\
2,00)\end{array}$ & $\begin{array}{c}<0,00 \\
1\end{array}$ \\
\hline Hipertensão & & & & & & & & & & & & \\
\hline Não & 1 & - & 1 & - & - & - & - & - & - & 1 & - & 1 \\
\hline Sim & 1,16 & $\begin{array}{c}(0,97- \\
1,40)\end{array}$ & 0,092 & - & - & - & - & - & - & 0,95 & $\begin{array}{c}(0,35- \\
2,53)\end{array}$ & 0,923 \\
\hline $\begin{array}{l}\text { Histórico de DCV } \\
\text { na família }\end{array}$ & & & & & & & & & & & & \\
\hline Não & 1 & - & 1 & 1 & - & 1 & 1 & - & 1 & 1 & - & 1 \\
\hline Sim & 1,07 & $\begin{array}{c}(0,99- \\
1,16)\end{array}$ & 0,050 & 1,22 & $\begin{array}{c}(0,85- \\
1,75)\end{array}$ & 0,259 & 1,07 & $\begin{array}{c}(1,00- \\
1,15)\end{array}$ & $\begin{array}{l}\mathbf{0 , 0} \\
\mathbf{3 3}\end{array}$ & 1,32 & $\begin{array}{c}(0,83- \\
2,09)\end{array}$ & 0,231 \\
\hline
\end{tabular}

IRR: Risco Relativo; IC: Intervalo de Confiança; DCV: Doença Cardiovascular.

Fonte: Dados da Pesquisa

Ainda, na análise ajustada, com relação ao desfecho $\mathrm{CP}$, ter ensino superior completo (IRR=1,14; IC=1,02-1,27), fumar (IRR=1,12; IC=1,03-1,21) e histórico de DCV na família $(\mathrm{IRR}=1,07 ; \mathrm{IC}=1,00-1,15)$ foram fatores de risco à $\mathrm{DCV}$ e ser do sexo feminino $\quad(\mathrm{IRR}=0,71 ; \quad \mathrm{IC}=0,66-0,76) \quad \mathrm{e}$ diabético $\quad(\mathrm{IRR}=0,62 ; \quad \mathrm{IC}=0,57-0,67)$ tiveram efeitos protetores e quanto ao indicador antropométrico RCQ, ser diabético (IRR=9,47; IC = 1,21-2,00), foi fator de risco à $\mathrm{DCV}$ e ser do sexo feminino $(\mathrm{IRR}=0,35$; IC $=0,21-0,58)$ e fazer uso de suplemento $\quad(\mathrm{IRR}=0,46 ; \quad \mathrm{IC}=0,26-0,79)$ foram fatores de proteção (Tabela 4).

\section{Discussão}

$\mathrm{Na}$ presente pesquisa investigamos os fatores associados a quatro indicadores antropométricos de risco cardiovascular (CC, RCEst, CP e RCQ) em praticantes de musculação. O primeiro desfecho estudado foi a $\mathrm{CC}$, apresentando na análise ajustada associação com sexo masculino, morar com companheiro, ter menor tempo de exercício físico, histórico de DCV na família e ser diabético. No entanto, fazer uso de suplemento apresentou fator de 
proteção para risco cardiovascular segundo o desfecho CC.

Segundo dados do estudo realizado com adultos praticantes de atividade física em uma academia de ginástica na cidade de Belo Horizonte ${ }^{21}$, os homens também apresentaram maior risco de desenvolver DCV que as mulheres, possivelmente devido os mesmos apresentarem mais gordura na região abdominal o que está associado a um maior risco cardiovascular. $^{22}$

Em relação ao estado civil, no presente estudo, viver com companheiro foi fator de risco para DCV de acordo com o indicador CC. Em pesquisa realizada com objetivo de determinar a prevalência de obesidade abdominal e identificar associação entre fatores socioeconômicos e estilo de vida em adultos atendidos numa clínica escola em São Luís - MA, observouse que obesidade abdominal diagnosticada pela $\mathrm{CC}$ foi associada a morar com o companheiro $(\mathrm{OR}=1,53 ; \mathrm{IC}=1,07-2,18$; $\mathrm{p}=0,017)^{23}$. Sugere-se que viver com companheiro possivelmente pode influenciar $\mathrm{o}$ aumento $\mathrm{da}$ obesidade abdominal devido às mudanças no comportamento social do casal, acarretando no aumento da frequência a restaurantes e lanchonetes, além do maior consumo de alimentos calóricos ${ }^{24}$.

Quanto à associação com indivíduos que praticavam exercício físico há menos tempo, observou-se que esses apresentavam maior risco de DCV. Em estudo realizado por Trapé et al. ${ }^{25}$ relacionou-se o Nível Habitual de Atividade Física (NHAF) e aptidão física com a saúde cardiovascular de adultos e idosos, e foi observado que $31,9 \%$ dos entrevistados foram classificados como ativos e evidenciou-se que os grupos de Índice de Aptidão Funcional Geral (IAFG), classificados como "bom" e "muito bom", apresentaram maior proporção de indivíduos com prática regular de exercícios físicos superior a seis meses, com destaque para um maior número que realizou a prática com supervisão $(p<0,0001)$. Além disso, os autores apontam que os grupos com IAFG mais elevados apresentaram melhores resultados em todas essas variáveis (IMC, HDL-c, TG, PAS e PAD) sugerindo uma relação forte entre o IAFG e as variáveis relacionadas aos fatores de risco para $\mathrm{DCV}$. Nesse caso, acredita-se que quanto maior a frequência e aptidão menor é o risco cardiovascular.

Em relação ao histórico de DCV na família, os dados deste estudo são semelhantes aos resultados obtidos por Silva $^{26}$ o qual encontrou associação significativa entre $\mathrm{CC}$ e variáveis história familiar de DCV $(p=0,012)$ e também um elevado percentual de entrevistados com histórico de DCV na família $(78,4 \%)$. Em outro estudo realizado, cujo objetivo foi avaliar o risco de acidentes cardiovasculares em praticantes de musculação, o histórico familiar foi observado como um dos principais fatores de risco, o qual, uma parcela dos participantes de musculação apresentou dados significantes para futuramente apresentar algum distúrbio metabólico ${ }^{27}$. Portanto, é importante que sejam investigados os dados familiares para que ocorra uma prevenção das DCV.

Os praticantes de musculação que eram diabéticos tiveram o risco aumentado para DCV, segundo a CC. Em estudo para verificar o perfil antropométrico de indivíduos que iniciaram a prática da musculação em uma academia de Goiânia ${ }^{28}$, verificou-se que os valores de CC podem predispor a pressão arterial elevada, diabetes mellitus, entre outros fatores de risco.

Vale ressaltar, entre as medidas antropométricas, a $\mathrm{CC}$ tem sido a mais utilizada para avaliar os riscos de DCV, uma vez que se associa ao acúmulo de tecido adiposo visceral ${ }^{29}$.

O segundo desfecho estudado foi a RCEst que tem sido indicada como um bom indicador antropométrico para identificar o risco de $\mathrm{DCV}^{30}$.

Segundo a análise ajustada, o sexo feminino foi fator de risco para DCV, 
segundo a RCEst. Estudo com 120 mulheres utilizou o IMC para verificar a correlação com os indicadores antropométricos de risco cardiovascular ${ }^{31}$, encontraram que $60,8 \%$ das mulheres avaliadas tinham risco para DCV por meio da RCEst, além disso, apontaram que todos os indicadores antropométricos avaliados se mostraram moderadamente correlacionados com o IMC, com exceção da RCEst que teve forte correlação.

Apresentar diabetes aumenta o risco de DCV, segundo a RCEst. Estudo realizado em pacientes diabéticos, em Vitória de Santo Antão-PE, dentre as variáveis que apresentaram maior razão de prevalência, a RCEst esteve presente, indicando que a obesidade abdominal infere na composição corporal do paciente diabético $^{32}$, confirmando o achado da presente pesquisa.

O terceiro desfecho estudado foi a $\mathrm{CP}$, pois é um marcador de medida de gordura subcutânea localizada na porção superior do corpo e tem demonstrado ser metabolicamente ativa, capaz de liberar ácidos graxos livres na circulação ${ }^{29}$. Desse modo, esse tipo de gordura pode favorecer o aparecimento de alterações metabólicas adversas $^{33}$, o que torna a $\mathrm{CP}$ uma medida importante para avaliação do risco cardiovascular.

Para o desfecho CP foram observados ser fator de risco o sexo feminino, escolaridade, fumo, histórico familiar de DCV e fator de proteção apresentar diabetes. Quanto ao risco ser maior no sexo feminino, esse resultado discorda do estudo de Santiago et al. ${ }^{35}$ no qual encontraram associação com o sexo masculino. Essa diferença de resultados pode ser explicada, pois no estudo de Santiago et al..$^{34}$ houve um maior percentual do sexo masculino, alta frequência de indivíduos sedentários e maior consumo de bebidas alcoólicas.

Apresentar maior escolaridade é fator de risco para DCV através da CP. Em busca na literatura não foram encontrados estudos que mostram essa associação.
Contudo, Lima $^{35}$ ao analisar a associação entre escolaridade e ocorrência de doenças crônicas em adultos de 62.986 domicílios, revelou que os indivíduos com menor escolaridade apresentaram maior chance de ter hipertensão arterial (OR:1,50; IC95\%: $1,30-1,73)$ e acidente vascular cerebral (OR: 2,76; IC95\%: 1,90-4,00).

Quanto à variável fumo, a literatura carece de estudos que tenham avaliado a associação entre a CP e ser tabagista. Entretanto, em estudo de base populacional, realizado com indivíduos de ambos os sexos, com idade de 20 a 59 anos, residentes na região metropolitana de Maringá Paraná, observou-se que a chance de ocorrência de infarto do miocárdio $(p=0,035 ; \quad O R=2,3 ; \quad I C=1,06-5,40) \quad$ e insuficiência cardíaca $(\mathrm{p}=0,028 ; \mathrm{OR}=2,3$; $\mathrm{IC}=1,09-4,94)$ foi maior em indivíduos tabagistas $^{36}$.

Possuir história familiar de DCV aumenta o risco de DCV por meio do indicador CP. Dados semelhantes ao resultado encontrado em pesquisa realizada com universitários da cidade de Salvador BA, cujo o objetivo foi conhecer a distribuição da gordura corporal e os fatores sociais de risco cardiovascular, dos 100 estudantes avaliados, $24 \%$ apresentaram CP superior a $34 \mathrm{~cm}$, e observou-se que os valores da medida da CP foi associado com o aumento do risco cardiovascular, além de revelar que as comorbidades mais prevalentes foram a história familiar de doença arterial coronariana seguida da obesidade $^{37}$.

Um achado que chamou a atenção no presente estudo foi o fato do praticante de musculação diabético ser fator protetor para a $\mathrm{CP}$, entretanto esse achado difere do encontrado por Pardo, Cabral e Haddad ${ }^{38}$. Em estudo, cujo objetivo foi avaliar a correlação da CP com resistência insulínica e com componentes da síndrome metabólica, os autores observaram correlação positiva entre $\mathrm{CP}$ e marcadores de resistência insulínica. Esse resultado pode ser explicado pelo aumento dos ácidos graxos livre que interferem no crescimento 
da produção de espécies reativas de oxigênio e ativação de células próinflamatórias como as citocinas, o que poderia resultar em resistência à insulina ${ }^{29}$.

O quarto desfecho foi a RCQ, esse índice tem se destacado como um indicador para obesidade abdominal e é fortemente relacionado a fatores de riscos cardiovasculares. $^{30}$

Para o desfecho RCQ foram encontradas associações com sexo feminino, ser diabético e consumir suplemento. Silva, Reis e Rodrigues ${ }^{39}$ ao analisarem o perfil nutricional dos servidores do Hospital Universitário de Montes Claros-MG, encontraram que o indicador RCQ indicou risco alterado de saúde para mulheres. Na mulher a gordura está localizada em maior quantidade nos quadris, culotes e nádegas, além das mulheres acumularem mais gordura para que os hormônios femininos funcionem melhor $^{40,41}$, provavelmente esse fato pode ter contribuído para o presente achado. Montalvão et al. $^{42}$ avaliaram a RCQ em mulheres praticantes de atividade física em academias e observaram que, aquelas com idade de 18 a 29 anos apresentaram RCQ baixa $(<0,71)$ e mulheres de 30 a 60 anos RCQ moderada $(0,72$ a 0,83$)$. Já, em investigação realizada com 214 servidores do Hospital Universitário da cidade de Petrolina-PE, a RCQ mostrou ser mais eficiente para detectar o risco de saúde em servidores do sexo masculino, o nível de correlação foi moderado para os homens e fraco para as mulheres ${ }^{43}$, resultado que difere do encontrado na presente pesquisa.

$\mathrm{O}$ risco em indivíduos diabéticos também foi observado no estudo de Oliveira et $\mathrm{al}^{44}$ ao estimarem a prevalência de sobrepeso e obesidade e determinarem a relação de diferentes indicadores antropométricos com os níveis glicêmicos de servidores da Universidade Federal de Viçosa em Minas Gerais. Observaram que os homens obtiveram correlação fraca com os níveis glicêmicos e os parâmetros analisados, entre eles a RCQ $(r=0,42)$ discordando do resultado encontrado na presente investigação.

O consumo de suplemento foi fator protetor para o risco de DCV para os desfechos estudados CC, RCEst e RCQ. Em pesquisa realizada com praticantes de musculação de uma academia na cidade de Varginha-MG, os autores evidenciaram que o consumo de suplemento termogênico, composto por cafeína, chá verde, capsaicinóides e citrus aurantium, mostrouse eficiente na redução de percentual de gordura corporal e circunferência abdominal dos praticantes de musculação ${ }^{45}$. Possivelmente esse resultado está ligado a maior oxidação dos ácidos graxos musculares, o que por sua vez poupa as reservas de carboidratos, retardando assim a fadiga e diminuindo a gordura corporal ${ }^{46}$.

Muraro e Saldanha ${ }^{47}$ salientam em sua pesquisa que o uso de termogênicos resulta em alteração nos parâmetros da composição corporal e taxas metabólicas, com diminuição significativas de peso, gordura corporal e IMC, porém, ressaltam que esses resultados foram encontrados em estudo que associou o uso de termogênicos em praticantes de exercício físico regular.

É importante ressaltar algumas limitações encontradas no desenvolvimento do presente estudo. Durante a coleta de dados não foi avaliado o nível de atividade física dos indivíduos e nem verificados os exames bioquímicos que caracterizam risco cardiovascular. Outra limitação a considerar refere-se ao delineamento transversal, que dificulta as análises de associação causal.

O ponto forte do presente estudo é o fato de trabalhar com uma amostra formada por praticantes de musculação, população na qual é pouco investigada quanto aos riscos cardiovascular, além da utilização dos quatro indicadores antropométricos (CC, RCEst, CP e RCQ). Esses indicadores vêm sendo bastante utilizados para avaliar os riscos de DCV. 
Brito AP, Rêgo AS, Lucian Sousa LMN, Ewerton FASL, Brasil GVS, Bragança MLBM, Silva FMAM, Barbosa JMA

\section{Conclusão}

Os dados do presente estudo mostram que há vários fatores que foram associados aos indicadores antropométricos de risco cardiovascular (CC, RCEst, CP e RCQ) em praticantes de musculação. Esses indicadores foram preditores na detecção do risco cardiovascular nesse público estudado. Portanto, praticar exercício físico não deve ser um único fator de profilaxia, outros hábitos deverão ser modificados para a diminuição do risco cardiovascular.

\section{Referências}

1. WHO. Global status report on noncommunicable diseases 2014. Geneva: World Health Organization, 2014.

2. Organização Pan-Americana de Saúde. Organização Mundial da Saúde. Doenças cardiovasculares. Brasilia, DF, Brasil: OPAS/OMS; 2017. [Internet] Disponível em:https://www.paho.org/bra/index.php?option=com_content\&view=article\&id=5253:doen cas-cardiovasculares\&Itemid=1096. Acesso em: 27 abril 2020.

3. Fábio Massalli. Mais de 289 mil pessoas morreram de doenças cardiovasculares em 2019. Agência Brasil, Brasília/DF, 24 set 2019. [Internet]. Disponível em: https://agenciabrasil.ebc.com.br/saude/noticia/2019-09/mais-de-289-mil-pessoas-morreramde-doencas-cardiovasculares-em-2019. Acesso em: 27 abril 2020.

4. Magalhães FJ, Mendonça LBA, Rebouças CBA, Lima FET, Custódio IL, Oliveira SC. Fatores de risco para doenças cardiovasculares em profissionais de enfermagem: estratégias de promoção da saúde. Rev. Bras. Enferm. 2014;67(3): 394-400.

5. Précoma DB, Oliveira GMM, Simão AF, Dutra OP, Coelho OR et al. Atualização da Diretriz de Prevenção Cardiovascular da Sociedade Brasileira de Cardiologia. Arquivos Brasileiros de Cardiologia. 2019;113(4):787-891.

6. Bensenor IM, Goulart AC, Santos IS, Lotufo PA. Prevalência de fatores de risco cardiovascular no mundo e no Brasil. Rev Soc Cardiol. 2019;29(1):18-24.

7. Cordovil YF, Almeida SS. Variáveis antropométricas e fatores de risco cardiovascular associados em Quilombolas Marajoaras. RBONE. 2018;12(71):406-15.

8. Caetano KA, Flores IA, Vilarinhos PAP, Fonseca DC, Martins ERT; Brandão HP et al. Indicadores Antropométricos e Risco Cardiovascular em Universitários: Um Mapeamento da Produção. Rev Cereus. 2018;10(1):111-29.

9. Pitanga FJG. Antropometria na avaliação da obesidade abdominal e risco coronariano. Rev Bras Cineantropom Desempenho Hum. 2011;13(3):238-41.

10. Carvalho CA, Fonseca PCA, Barbosa JB, Machado SP, Santos AM, Silva AAM. Associação entre fatores de risco cardiovascular e indicadores antropométricos de obesidade em universitários de São Luís, Maranhão, Brasil. Ciênc Saúde Colet. 2015;20(2):479-90.

11. Pohl HH, Arnold EF, Dummel KL, Cerentini TM, Reute EM, Reckziegel MB. Indicadores antropométricos e fatores de risco cardiovascular em trabalhadores rurais. Rev Bras Med Esporte. 2018;24(1):64-8.

12. Preis SR, Massaro JM, Hoffmann U, D'Agostino RB Sr, Levy D, Robins SJ, et al. Neck circumference as a novel measure of cardiometabolic risk: the Framingham.

13. IHRSA. The 2019 IHRSA Global Report: The state of the health club industry. Boston: IHRSA; 2019. [Internet]. Disponível em: https://www.ihrsa.org/publications/the-2019-ihrsaglobal-report/ Acesso em: 27 abril 2020.

14. Hilgenberg FE. Prevalência de fatores de risco cardiovascular em cadetes da academia da força aérea brasileira. 97F. [Dissertação Mestrado Nutrição e Saúde] - Programa de PósGraduação em Nutrição e Saúde, da Faculdade de Nutrição da Universidade Federal de Goiás, 2013.

15. Guedes DP. Recursos antropométricos para análise da composição corporal. Rev Bras Educ Fís Esp. 2006;20:115-19. 
16. WHO, World Health Organization. Obesity. Preventing and Managing the Global Epidemic. Report of a WHO Consultation on Obesity, Geneva: World Health Organization, p. 107-158, 1997.

17. WHO, World Health Organization. Waist circumference and waist-hip ratio. Report of a WHO Expert Consultation. Geneva: World Health Organization, 2008.

18. Ben-Noun L, Sohar E, Laor A. Neck circumference as a simple screening measure for identifying overweight and obese patients. Obes Res. 2001;9(8):470-7.

19. Pitanga FJG, Lessa I. Razão cintura-estatura como discriminador do risco coronariano de adultos. Rev Assoc Med Bras. 2006;52(3):157-61.

20. World Health Organization. Physical status: The use and interpretation of anthropometry. WHO Technical Report Series, n. 854, 1995.

21. Dinardi RR, Dinardi LR, Soares DD. Análise de parâmetros de risco coronariano em adultos praticantes de atividade física em uma academia de ginástica localizada em um bairro de alto padrão socioeconômico na cidade de Belo Horizonte, MG. Revista Digital. Buenos Aires. 2009. [Internet]. Disponível em: https://www.efdeportes.com/efd139/risco-coronariano-emadultos-praticantes-de-atividade-fisica.htm

22. Barroso TA, Marins LB, Alves R, Gonçalves CS, Barroso SG, Rocha GS. Associação Entre a Obesidade Central e a Incidência de Doenças e Fatores de Risco Cardiovascular. Int $\mathbf{J}$ Cardiovasc Sci. 2017;30(5)416-24.

23. Sabóia RS, Araújo AP, Barbosa JMA, Galvão CRP, Cruvel JMS, Ferreira SCN. Obesidade abdominal e fatores associados em adultos atendidos em uma clínica escola. Rev Bras Promoç Saúde. 2016;29(2):259-67.

24. Santiago JCS, Moreira TMM, Florêncio RS. Associação entre excesso de peso e características de adultos jovens escolares: subsídio ao cuidado de enfermagem. Rev Latino Am Enfermagem. 2015;23(2):250-58.

25. Trapé AA, Lizzi EASS, Jacomini AM, Hott SC, Júnior CRB, Zago AS. Aptidão física e nível habitual de atividade física associados à saúde cardiovascular em adultos e idosos. Medicina. 2015;48(5):457-66.

26. Silva VC. Análise dos fatores de risco para doença cardiovascular em crianças escolares de 5 a 9 anos procedentes de escolas públicas da região central da cidade de Fortaleza-CearáBrasil. 2017.182F. [Tese Doutorado em Ciências] - Programa de Pediatria, Faculdade de Medicina da Universidade de São Paulo, São Paulo, 2017.

27. Rosa TS, Leite MO. Risco de infarto agudo do miocárdio em praticantes de musculação de uma academia da cidade de Itatiaia-RJ. RBNE. 2014;8(44): 92-102.

28. Vieira IP, Machado JHJO, Schmidt A. Perfil antropométrico de iniciantes de musculação de uma academia de ginástica em Goiânia. Rev Digital Buenos Aires. 2013. [Internet]. Disponível em: https://www.efdeportes.com/efd176/perfil-antropometrico-de-iniciantes-demusculacao.htm

29. Barbosa PS, Santos RP, Mendonça JLS, Rocha VS. Circunferência do pescoço e sua associação com parâmetros antropométricos de adiposidade corporal em adultos. Brasben J. 2017;32(4):315-20.

30. Ulguim F, Renner JDP, Pohl HH. Risco para doenças cardiovasculares, em trabalhadores de um hospital de ensino do RS: Análise a partir da circunferência de cintura e relação cinturaquadril. Anais do Seminário Científico do Programa de Pós-Graduação em Promoção da Saúde da UNISC. 2015;1(1).

31. Moraes KDM, Araújo AP, Santos AF, Barbosa JMA, Martins MLB. Correlação entre o índice de massa corporal e indicadores antropométricos de risco cardiovascular em mulheres. Rev Pesq Saúde. 2015;16(3):175-81.

32. Melo MA, Tassitano RM, Petribú MMV, Santos EMC, Santana RA, Campos FAZ. Proteína C-reativa como biomarcador inflamatório e fatores associados em diabéticos tipo 2 atendidos na rede pública de saúde. Nutr Clín Diet Hosp. 2016;36(2):83-95. 
33. Selvan C, Dutta D, Thukral A, Nargis T, Kumar M, Mukhopadhyay S, Chowdhury S. Neck height ratio is an important predictor of metabolic syndrome among Asian Indians. Indian $\mathrm{J}$ Endocrinol Metab. 2016;20(6):831-37.

34. Santiago ERC, Dourado KF, Petribú MMV, Andrade MIS, Barbosa LS, Santos CM. Circunferência do pescoço como indicador de risco cardiovascular em pacientes renais crônicos em hemodiálise. Nutr Clin Diet Hosp. 2017;37(1):41-8.

35. Lima RN. Associação da escolaridade com a prevalência de doenças crônicas e a limitação das atividades diárias causadas por doenças crônicas: estudo de base populacional no Brasil, 2013.2016.125F. [Dissertação Mestrado em Saúde Coletiva] - Centro de Ciências da Saúde, Universidade Federal de Santa Catarina, Florianópolis, 2016.

36. Teston EF, Cecilio HPM, Santos AL, Arruda GO, Radovanovic CAT, Marcon SS. Fatores associados às doenças cardiovasculares em adultos. Medicina. 2016;49(2):95-102.

37. Rezende IFB, Toledo DVC, Silva LR. Distribuição de gordura corporal da região superior do corpo e dos fatores sociais de risco cardiovascular em estudantes do curso de nutrição em Salvador/BA. Rev Ciênc Med Biol. 2015;14(3):355-59.

38. Pardo IMC, Cabral EB, Haddad JR. Circunferência do pescoço: nova medida antropométrica para rastreamento da síndrome metabólica em adolescentes. Arq Catarin Med. 2019;48(2):21-33.

39. Silva AF, Reis FKR, Rodrigues VD. Perfil nutricional de funcionários do Hospital Público de Montes Claros/MG. Rev Multitexto. 2016;4(1):82-8.

40. Mendonça LS, Moreira JAR. A influência dos hormônios leptina e insulina na gordura localizada. Rev Científica FHO. 2015;3(2):47-56.

41. Silva JP, Souza FS, Assis IB, Pereira LP. Ultracavitação para gordura localizada - Revisão de literatura. Rev Saúde em Foco. 2018; 10:702-10.

42. Montalvão DMA, Sá AC, Paixão PLR, Machado ALA, Navarro ACA. Correlação do risco de doenças cardiovascular e relação cintura quadril (RCQ) em mulheres praticantes de atividade física. RBONE - Revista Brasileira De Obesidade, Nutrição E Emagrecimento. 2008;2(10):324-27.

43. Maduro PA, Nascimento MM, Araújo IS, Barbosa LF, Pereira LGD. Análise do estado nutricional e nível de atividade física dos servidores do hospital universitário de PetrolinaPE. RBNE. 2017;11(67) (Suppl1):856-67.

44. Oliveira RAR, Amorim PRS, Moreira OC, Amorim W, Costa EG, Marins JCB. Relação de indicadores antropométricos com glicemia entre servidores universitários. Rev Ciênc Méd. 2015;24(1):19-28.

45. Silva LCJ, Pereira EAA, Machado GJ. Efeito de um suplemento termogênico na composição corporal de praticantes de musculação. RBNE. 2019;13(80):534-42.

46. Peçanha AMM, Friferi R, Filho JNS. Suplementos termogênicos: evidências sobre a sua eficácia na redução da gordura corporal. RBNE. 2017;11(65):544-53.

47. Muraro CR, Saldanha RP. Uma revisão de literatura sobre o uso de termogênicos e seus efeitos no organismo. Rev Perspect Ci Saúde. 2016;1(1):85-96.

\section{Como citar este artigo:}

Brito AP, Rêgo AS, Lucian Sousa LMN, Ewerton FASL, Brasil GVS, Bragança MLBM, Silva FMAM, Barbosa JMA. Risco cardiovascular em praticantes de musculação em academias de São Luís - MA. Rev. Aten. Saúde. 2021; 19(67): 92-106. 